\title{
INVARIANT MEASURES IN GROUPS WHICH ARE NOT LOCALLY COMPACT
}

BY

\author{
JOHN C. OXTOBY
}

1. Introduction. The problem of measure in groups is the problem of defining a left-invariant measure, with specified properties, in a given group. It is a special case of the general problem of defining a measure in a set with a given congruence relation( $(1)$. In a topological group it is natural to require that the measure be defined at least for all Borel sets, and therefore to consider Borel measures. By a Borel measure we understand a countably additive non-negative set function defined for all (and only for) Borel sets. It may assume the value $+\infty$ but is required to be finite and positive for at least one set. In addition, we require always that points have measure zero. A Borel measure $m$ in a group $G$ is left-invariant if $m(x A)=m(A)$ for every Borel set $A \subset G$ and every element $x \in G$. The present paper is devoted to a study of the existence and possible properties of such measures.

In locally compact separable groups the problem of measure, with the added requirement that the measure be locally finite, was solved by Haar [7], as is well known. Moreover, Haar's measure is unique in the sense that (except for a constant factor) it is the only locally finite left-invariant Borel measure in such a group (see von Neumann [17]). However, a great variety of measures which are not locally finite always exist, as we shall see.

The present paper contains a solution of the problem of measure in complete separable metric groups. (By a metric group we mean a topological group with a metrizable topology. It is complete if it is a complete space with respect to some metric.) It will be shown that there exists a left-invariant Borel measure in any such group, unless the group is countable, but that a locally finite measure is possible only if the group is locally compact. This makes it possible to state Haar's theorem in the following generalized form. In a complete separable metric group there exists a locally finite left-invariant Borel measure if and only if the group is locally compact and dense in itself.

The problem of measure in complete metric groups is solved here by a construction which differs considerably from Haar's and makes no use of his result, though it utilizes only fairly standard techniques. Other constructions, based partly on Haar's theorem, are discussed, but it is shown by examples that these methods are not universally applicable. Weil's theory [24, Ap-

Presented to the Society, September 17, 1945; received by the editors November 12, 1945.

(1) See, for example, Banach [2], Loomis [15], and Maharam [16]. Numbers in brackets refer to the Bibliography at the end of the paper. 
pendix I] of measure in groups is considered in some detail, with a view to evaluating its significance for the problem of measure under consideration. It is believed that this investigation throws considerable light on the limitations of Weil's theory by exhibiting the possibility of measures that fail to satisfy his postulates, and by showing that in some cases it is impossible for a Borel measure to satisfy them.

Finally, a number of examples are considered in the light of the general theory. In particular, it is shown that in the additive group of real numbers one can introduce a large variety of different topologies, with invariant measures that range from the most regular to the most pathological.

2. The impossibility of a locally finite measure. The generalized form of Haar's theorem stated above is an immediate consequence of the following theorem, the proof of which is due to S. Ulam $\left({ }^{2}\right)$.

Theorem 1. Let $G$ be any complete separable metric group which is not locally compact, and let $m$ be any left-invariant Borel measure in $G$. Then every neighborhood contains non-denumerably many disjoint mutually congruent sets of equal finite positive measure.

Two sets $A$ and $B$ are said to be congruent if there exists an elemènt $x \in G$ such that $B=x A$.

We first establish a lemma which belongs to the general theory of measure in complete separable spaces and has nothing specifically to do with groups.

LeMMA 1. Let $m$ be any Borel measure in a complete separable metric space. The measure of any Borel set $B$ with finite positive measure is equal to the least upper bound of the measures of compact subsets of $B$.

This result can be proved on the basis of a theorem of Kuratowski [14, p. 231], which asserts that any Borel subset $B$ of a complete separable space can be represented as a one-to-one continuous image of another complete separable space $E$ by a mapping $f$ whose inverse is Borel measurable. Such a mapping $f$ defines a finite Borel measure $\mu$ in $E$, namely the measure $\mu A=m(f A)$. But if $\mu$ is any finite Borel measure in a complete separable space $E$, then for each positive number $\epsilon$ there exists $\left({ }^{3}\right)$ a compact set $C \subset E$ such that $\mu C \geqq \mu E-\epsilon$. Hence $m(f C) \geqq m B-\epsilon$, and since $f C$ is a compact subset of $B$ the lemma is proved.

Now, to prove Theorem 1, consider the given topological group $G$ with left-invariant measure $m$, and let $U$ be an arbitrary neighborhood. By hypothesis, there exists a Borel set $B$ with finite positive measure. Since the space is separable, a countable number of sets of the form $x U$ suffice to cover

$\left({ }^{2}\right)$ The author wishes to thank Professor Ulam for permission to use this result, and also for numerous helpful suggestions and remarks.

( 3 See $[19$, p. 561 , footnote 3$]$. The paper there referred to was never published, but the proof is entirely contained in this footnote. 
$G$. Consequently at least one of these sets, say $a U$, intersects $B$ in a set of positive measure. Therefore $a^{-1} B \cap U$ is a Borel subset of $U$ with finite positive measure. By Lemma 1, this contains a compact set $C$ with $0<m C<\infty$.

Consider the subgroup $G_{1}$ generated by the set $C$. Observe first that the group product $C_{1} C_{2}$ and the set $C_{1}^{-1}$ of inverses of elements of $C_{1}$ are both compact if the sets $C_{1}$ and $C_{2}$ are compact. Starting with the set $C$, form the sets $C_{1}=C \cup C^{-1}$ and $C_{n+1}=C_{n} C_{n}, n \geqq 1$. All these sets are compact, and their union $\mathrm{U}_{n} C_{n}$ consists of all finite products of elements of $C$ or $C^{-1}$ and is therefore equal to $G_{1}$. Now, in a non locally compact group any compact set is nowhere dense. Consequently $G_{1}$ is of first category in $G$, and likewise each of its left-cosets. Let $V$ be a neighborhood of unity so small that $V C \subset U$. The leftcosets of $G_{1}$ corresponding to elements of $V$ cover $V$. Since $V$ is of second category, and the left-cosets of $G_{1}$ are mutually disjoint, $V$ must contain nondenumerably many elements belonging to distinct left-cosets of $G_{1}$. Each of these cosets contains a set congruent to $C$ and contained in $U$. Hence $U$ contains non-denumerably many disjoint sets of the form $x C$, which proves Theorem 1.

By a refinement of the foregoing reasoning we can prove the following stronger theorem.

THEOREM 2. Under the same assumptions as Theorem 1, any neighborhood contains a compact set which is the union of non-denumerably many disjoint mutually congruent compact sets of equal finite positive measure.

To prove this, let $U, C$, and $V$ be defined as in the proof of Theorem 1 . Then $C C^{-1}$ is a compact and nowhere dense set. It is therefore possible to find a sequence $\left\{a_{n}\right\}$ of elements of $V$ such that $a_{n} \rightarrow e$ and $a_{n} \notin C C^{-1}$. Consider the countable set of all products of the form

$$
a_{1}^{i_{1}} a_{2}^{i_{2}} \cdots a_{n}^{i_{n}}
$$$$
\left(i_{1}, \cdots, i_{n}=0,1 ; n=1,2, \cdots\right)
$$

and let $\Gamma$ denote the closure of this set. If the sequence $\left\{a_{n}\right\}$ tends to $e$ suffciently rapidly, we shall show that the set $\Gamma$ is homeomorphic to the Cantor set, that it is contained in $V$, and that $\Gamma^{-1} \Gamma \cap C C^{-1}$ contains only the element $e$. This last property implies at once that if $x$ and $y$ are distinct elements of $\Gamma$, then the sets $x C$ and $y C$ are disjoint. Consequently $\Gamma C$ is a compact subset of $U$ that fulfills the requirements of Theorem 2.

In order that the set $\Gamma$ shall have the desired properties, it is sufficient to start with any sequence $\left\{\bar{a}_{n}\right\}$ such that $\bar{a}_{n} \rightarrow e$ and $\bar{a}_{n} \notin C C^{-1}$, and take for $\left\{a_{n}\right\}$ the subsequence defined inductively as follows. Let $a_{1}$ be the first element of the sequence $\left\{\bar{a}_{n}\right\}$ such that the sphere about $e$ with radius $2 \rho\left(e, a_{1}\right)$ is contained in $V$. If $a_{1}, a_{2}, \cdots, a_{n}$ have already been defined, let $A_{n}$ denote the finite set consisting of the elements of the form (2.1) for fixed $n$, let $d_{n}$ denote the minimum distance between distinct points of $A_{n}$, and let 
$4 \delta_{n}$ denote the smallest of the three positive numbers $\rho\left(a_{n}, C C^{-1}\right), \rho\left(a_{n}^{-1}, C C^{-1}\right)$, and $d_{n}$. Then define $a_{n+1}$ as the first element of the sequence $\left\{\bar{a}_{n}\right\}$ such that each of the distances $\rho\left(a, a a_{n+1}\right), \rho\left(a, a_{n+1}{ }^{-1} a\right), \rho\left(a, a_{n+1}{ }^{-1} a a_{n+1}\right)$ is less than $\delta_{n}$ for every $a \in A_{n}{ }^{-1} A_{n}$. Such an element exists, since any element sufficiently near $e$ fulfills these conditions. To verify that the set $\Gamma$ determined by the sequence $\left\{a_{n}\right\}$ has the required properties, note first that since $e \in A_{n}{ }^{-1} A_{n}$ we have $\rho\left(e, a_{n+1}\right)<\delta_{n}, d_{n+1} \leqq \rho\left(e, a_{n+1}\right)$, and therefore $\delta_{n+1}<\delta_{n} / 4$. It is easy to see that the sequence of elements $x_{n}$ of the form (2.1) corresponding to any sequence $\left\{i_{n}\right\}\left(i_{n}=0,1\right)$ converges to an element $x$ in $\Gamma$, since $\rho\left(x_{n}, x_{n+p}\right)$ $<\delta_{n}+\delta_{n+1}+\cdots+\delta_{n+p-1}<(4 / 3) \delta_{n}$. Moreover, if $\left\{i_{n}\right\}$ and $\left\{j_{n}\right\}$ are distinct sequences, and $k$ is the first index for which $i_{k} \neq j_{k}$, the corresponding points $x$ and $y$ in $\Gamma$ are separated by a distance at least equal to $(4 / 3) \delta_{k}$, as the following calculation shows: $4 \delta_{k} \leqq d_{k} \leqq \rho\left(x_{k}, y_{k}\right) \leqq \rho\left(x_{k}, x\right)+\rho(x, y)+\rho\left(y, y_{k}\right)$ $\leqq(4 / 3) \delta_{k}+\rho(x, y)+(4 / 3) \delta_{k}$. It follows that the elements of $\Gamma$ are in oneto-one correspondence with the totality of sequences $\left\{i_{n}\right\}$, and an elementary argument then shows that the function $f(x)=\sum_{1}^{\infty} 2 i_{n} / 3^{n}$ effects a homeomorphism between $\Gamma$ and the Cantor ternary set. To show that $\Gamma \subset V$, it suffices to note that the distance from $e$ to any point of $A_{n}$ is less than $\rho\left(e, a_{1}\right)+\delta_{1}$ $+\cdots+\delta_{n-1}<(4 / 3) \rho\left(e, a_{1}\right)$. Finally, any element $z \in \Gamma^{-1} \Gamma$ is the limit of a sequence $\left\{b_{n}\right\}, b_{n} \in A_{n}^{-1} A_{n}$, and if $z \neq e$ we have $b_{k}=a_{k}^{ \pm 1}$ for some index $k$. Hence $\rho\left(b_{k}, z\right) \leqq \delta_{k}+\delta_{k+1}+\cdots \leqq(4 / 3) \delta_{k} \leqq(1 / 3) \rho\left(b_{k}, C C^{-1}\right)$, and so $z \notin C C^{-1}$. Therefore $\Gamma^{-1} \Gamma \cap C C^{-1}=e$. This completes the proof of Theorem 2 .

It may be noted that Theorem 1 does not necessarily hold for (improper) Borel measures that assume only the values 0 and $+\infty$, even when the word "finite" is omitted from the conclusion. We may introduce such a measure by defining $m A$ equal to 0 or $+\infty$ according as the Borel set $A$ is of first or second category. This measure is countably additive, left-invariant, and zero for points. But since any Borel set has the property of Baire, any Borel set of second category contains a sphere minus a set of first category (see, for example, Kuratowski $[14, \S 11])$. It follows that in a separable space any family of disjoint Borel sets of second category is denumerable, and so Theorem 1 fails to hold for this measure.

3. The problem of measure. Let $G$ be a complete metric group which is dense in itself. We may consider the following problems:

Problem 1. To define in $G$ a left-invariant Borel measure which is zero for points.

Problem 2. To define in $G$ a left-invariant Borel measure which is not only zero for points but also satisfies one or more of the following conditions:

(3.1) Some neighborhood has finite measure. (Such a measure is called locally finite.)

(3.2) The measure of any measurable set is equal to the lower bound of the measures of open sets that contain it.

(3.3) Every compact set has finite measure. 
(3.4) Any measurable set can be covered by a sequence of compact sets.

(3.5) Any measurable set can be represented as the union of countably many sets of finite measure.

If $G$ is a locally compact separable metric group, it is well known that Haar's measure has all five of the properties enumerated in Problem 2. However, Theorems 1 and 2 show that if $G$ is separable but not locally compact then no Borel measure can satisfy any of these conditions; in other words, Problem 2 is soluble only if the group is locally compact.

In case $G$ is locally compact but not separable, it is customary to consider Haar's measure as defined only for Borel sets which can be covered by countably many compact sets or, equivalently, which satisfy condition (3.5). In such a group Haar's measure is therefore not strictly a Borel measure. However, it can always be extended to a Borel measure, most simply by defining the measure of any Borel set as the least upper bound of the measures of its Haar measurable subsets. But other extensions are also possible (see $\$ 7$ ) and so it is desirable to limit the domain of Haar's measure in the above manner in order to preserve the theorem of uniqueness. On the other hand, from any measure $m$ one can derive a measure with the property (3.5) simply by contracting its domain to those sets which satisfy this condition. (It is easy to verify that the family of sets with property (3.5) is a Borel field, that is, that it contains the difference of any two and the union and intersection of any sequence of its members.) Since we shall have frequent occasion to refer to this remark, it is convenient to introduce the following definitions, which are suggested by the usual $\sigma$-terminology.

Definition 1. A measure $m$ (in any space) is called $\sigma$-finite if each set in its domain can be represented as the union of countably many sets of finite measure.

DEFINITION 2. The measure derived from a measure $m$ by limiting its domain to the least Borel field that includes all sets of finite measure is called the $\sigma$-finite contraction of $m$.

Thus Theorems 1 and 2 and Lemma 1 imply that the domain of the $\sigma$-finite contraction of any left-invariant Borel measure in a complete separable non locally compact group includes no open sets (except the void set) and includes some but not all compact sets.

In $\$ 9$ we shall consider another kind of condition that might be added to Problem 1, and shall find that it too renders the problem insoluble in general. It therefore appears that for general complete metric groups (separable or not) Problem 1 demands about as much as is possible for a Borel measure, and it is therefore of interest to show that this problem can be solved.

4. Construction of an invariant measure. We proceed to give a general solution of Problem 1. Other constructions, applicable to special classes of groups, will be considered in $\S 8$. 
THEOREM 3. In any complete metric group which is dense in itself there exists a left-invariant Borel measure.

We shall prove this theorem first in the separable case. The generalization to non-separable groups will be made in $\$ 7$.

The first step in the construction consists in the introduction of a leftinvariant metric in $G$. Kakutani [12] has shown that any topological group that satisfies the first countability axiom is metrizable, and that the metric can be taken to be left-invariant, that is, such that $\rho(z x, z y)=\rho(x, y)$ for all $x, y$, and $z$ in the group. Consequently, any metric group can be remetrized with a topologically equivalent left-invariant metric. We suppose this done, and for brevity denote the distance between $x$ and $y$ in the new metric simply by $(x, y)$. It is necessary to allow for the possibility that $G$ may be incomplete with respect to the new metric (cf. §6), but this introduces no serious complication.

We first describe a very general type of measure construction (cf. Hausdorff [10]) and then make the necessary specializations. Let $U_{1}, U_{2}, \cdots$ be a sequence of non-void open sets in $G$ whose diameters tend to zero. Let $w_{1}, w_{2}, \ldots$ be a sequence of finite positive numbers tending to zero. Let $\mathfrak{U}$ denote the family of sets in $G$ congruent to one of the sets $U_{n}$. Consider any set $A \subset G$, and any covering of $A$ by a finite or infinite sequence of sets of the family $\mathfrak{u}$, say $A \subset \bigcup_{i} b_{i} U_{n_{i}}$. Form the sum $\sum_{i} w_{n_{i}}$ and let $L_{r}(A)$ denote the lower bound of these sums for all covering sequences of sets $\mathfrak{U}$ with diameters less than $r$. (Such coverings always exist, indeed $A$ can be covered by a sequence of sets congruent to $U_{n}$, for any fixed $n$, since the space is separable.) It is evident that as $r$ decreases, $L_{r}(A)$ will not decrease. Hence the limit $m^{*} A=\lim _{r \rightarrow 0} L_{r}(A)$ exists or is equal to $+\infty$.

From this definition it is an easy matter to verify that the following conditions are satisfied:

(4.1) $0 \leqq m^{*} A \leqq+\infty$.

(4.2) $m^{*} A \leqq m^{*} B$ if $A \subset B$.

(4.3) $m^{*} \cup_{n} A_{n} \leqq \sum_{n} m^{*} A_{n}$ for any sequence $A_{n}$.

(4.4) $m^{*}(A \cup B)=m^{*} A+m^{*} B$ if $A$ and $B$ are separated by a positive distance.

(4.5) $m^{*}(x A)=m^{*} A$ for arbitrary $x \in G$ and $A \subset G$.

(4.6) $m^{*} A=0$ if $A$ contains only one point.

(4.7) $m^{*} A=\inf m^{*} B$ for all $G_{\delta}$ sets $B$ which contain $A$.

(Condition (4.7) follows from the fact that any set is contained in a $G_{\delta}$ set having the same outer measure, since only open coverings are employed.) It follows that $m^{*} A$ is a regular outer measure in the sense of Caratheodory [4, p. 238] (provided it is finite and positive for at least one set, see below) and therefore defines a left-invariant measure in $G$. In view of (4.7), this measure is the complete extension of a Borel measure $m$. We shall say that the 
Borel measure $m$ is the measure generated by the measuring system $\left\{U_{n}, w_{n}\right\}$.

We come now to the crucial point in the construction. We need to show that it is always possible to find a measuring system $\left\{U_{n}, w_{n}\right\}$ which generates a measure $m$ such that $0<m A<+\infty$ for at least one set. It is clear that not every measuring system will do this. For example, in the additive group of real numbers, if $U_{n}$ is an interval of length $1 / n$, and we take $w_{n}=n^{-2}$, every set will have measure zero. To secure a non-trivial measure it is therefore necessary to choose the measuring system with some care.

To this end, we shall construct in $G$ a family of sets $C\left(n ; i_{1}, \cdots, i_{n}\right)$ $\left(i_{1}, \cdots, i_{n}=0,1 ; n=1,2, \cdots\right)$ with the following properties. The sets $C\left(n ; i_{1}, \cdots, i_{n}\right)$ with primary index $n$ will be said to be of rank $n$.

(i) $C\left(n ; i_{1}, \cdots, i_{n}\right)$ is compact.

(ii) $C\left(n ; i_{1}, \cdots, i_{n}\right)=C\left(n+1 ; i_{1}, \cdots, i_{n}, 0\right) \cup C\left(n+1 ; i_{1}, \cdots, i_{n}, 1\right)$.

(iii) The $2^{n}$ sets of rank $n$ are mutually congruent.

(iv) The diameter $d_{n}$ of the sets of rank $n$ tends to zero as $n \rightarrow \infty$.

(v) Any two sets of rank $n$ are separated by a distance greater than $d_{n}$.

Define $C=C(1 ; 0) \cup C(1 ; 1)$. Then $C=\bigcup_{i_{1}} \ldots i_{n} C\left(n ; i_{1}, \cdots, i_{n}\right)$ for each $n$.

It may be remarked that the family of sets $C\left(n ; i_{1}, \cdots, i_{n}\right)$ evidently represent $C$ as a dyadic discontinuum (see Hausdorff $[11$, p. 134]), and therefore $C$ is homeomorphic to the Cantor set.

To construct such a family of sets, note first that since $G$ is dense in itself we can choose a sequence of points $x_{n}$ tending to the unity element $e$, such that

$$
0<\left(e, x_{n+1}\right) \leqq\left(e, x_{n}\right) / 9
$$

$$
(n=1,2, \cdots) \text {. }
$$

Define $a_{i_{1}} \cdots i_{n}=x_{1}{ }^{i_{1}} x_{2}{ }^{i_{2}} \cdots x_{n}{ }^{i_{n}}\left(i_{1}, \cdots, i_{n}=0,1\right)$. Let $A\left(n ; i_{1}, \cdots, i_{n}\right)$ denote the set of all points $a_{i_{1}} \ldots i_{n+p}, p \geqq 0$, whose first $n$ indices are equal to $i_{1}, i_{2}, \cdots, i_{n}$, and define $C\left(n ; i_{1}, \cdots, i_{n}\right)$ as the closure of the countable set $A\left(n ; i_{1}, \cdots, i_{n}\right)$. Evidently we have $A\left(n ; i_{1}, \cdots, i_{n}\right)=A\left(n+1 ; i_{1}, \cdots, i_{n}, 0\right)$ $\cup A\left(n+1 ; i_{1}, \cdots, i_{n}, 1\right)$ and $A\left(n ; i_{1}, \cdots, i_{n}\right)=a_{i_{1}} \ldots i_{n} A(n ; 0, \cdots, 0)$. Properties (ii) and (iii) follow at once by taking closures. Furthermore, the diameter $d_{n}$ of the sets $C\left(n ; i_{1}, \cdots, i_{n}\right)$ of rank $n$ is given by $d_{n}=\operatorname{diam} A(n ; 0, \cdots, 0)$. We proceed to obtain an estimate of $d_{n}$.

Note that the left-invariance of the metric implies that for arbitrary elements $y, y_{1}$, and $y_{2}$ in $G$ we have $\left(e, y_{1} y_{2}\right) \leqq\left(e, y_{1}\right)+\left(y_{1}, y_{1} y_{2}\right)=\left(e, y_{1}\right)+\left(e, y_{2}\right)$. Also $(e, y)=\left(y^{-1}, e\right)=\left(e, y^{-1}\right)$, so that $\left(e, y^{k}\right) \leqq(e, y)$ if $k=0, \pm 1$. A simple induction then shows that

$$
\left(e, y_{1}^{k_{1}} y_{2}^{k_{2}} \cdots y_{n}^{k_{n}}\right) \leqq\left(e, y_{1}\right)+\left(e, y_{2}\right)+\cdots+\left(e, y_{n}\right)
$$

for arbitrary $y_{1}, y_{2}, \cdots, y_{n}$ in $G$ and exponents $k_{1}, \cdots, k_{n}$ equal to 0 or \pm 1 .

Now consider any two points of $A(n ; 0, \cdots, 0)$. These may be written $x_{n+1}{ }^{i_{1}} \cdots x_{n+p} i_{p}$ and $x_{n+1}^{i_{1}} \cdots x_{n+p} j_{p}$, where the exponents $i_{k}$ and $j_{k}$ are either 0 or 1 . The distance between these points can be estimated as follows. 


$$
\begin{aligned}
\left(x_{n+1}^{i_{1}} \cdots x_{n+p}^{i_{p}}, x_{n+1}^{j_{1}} \cdots x_{n+p}^{j_{p}}\right) & =\left(e, x_{n+p}^{-i_{p}} \cdots x_{n+1}^{-i_{1}} x_{n+1}^{j_{1}} \cdots x_{n+p}^{j_{p}}\right) \\
& \leqq 2\left[\left(e, x_{n+1}\right)+\left(e, x_{n+2}\right)+\cdots+\left(e, x_{n+p}\right)\right] \\
& \leqq \frac{2}{9}\left(e, x_{n}\right)+\frac{2}{9^{2}}\left(e, x_{n}\right)+\cdots+\frac{2}{9^{p}}\left(e, x_{n}\right) \\
& <\left(e, x_{n}\right) / 4 .
\end{aligned}
$$

This being true for any two points of $A(n ; 0, \cdots, 0)$ it follows that $d_{n} \leqq\left(e, x_{n}\right) / 4$. On the other hand, $e$ and $x_{n+1}$ are both contained in $A(n ; 0, \cdots, 0)$, and so $\left(e, x_{n+1}\right) \leqq d_{n}$. Consequently $d_{n+1} \leqq\left(e, x_{n+1}\right) / 4 \leqq d_{n} / 4$, and so $d_{n+1} \leqq d_{n} / 4, n=1,2, \ldots$. It follows, in particular, that (iv) is satisfied.

Next consider any two distinct sets $A\left(n ; i_{1}, \cdots, i_{n}\right)$ and $A\left(n ; j_{1}, \cdots, j_{n}\right)$ and any two representative points of these sets. These may be written $a_{i_{1}} \ldots i_{p}$ and $a_{j_{1}} \ldots i_{p}$, where $p \geqq n$. Suppose their indices differ first in the $k$ th place. Then $k \leqq n$ and we may assume $i_{k}=0$ and $j_{k}=1$. Let $u=x_{k+1}{ }^{i_{k+1}} \cdots x_{p}{ }^{i_{p}}$ and $v=x_{k} x_{k+1}{ }^{j_{k+1}} \cdots x_{p}^{i_{p}}$. Then $\left(a_{i_{1}} \cdots i_{p}, a_{j_{1}} \cdots j_{p}\right)=(u, v)$ and

$$
\begin{aligned}
\left(e, x_{k}\right) & \leqq(e, u)+(u, v)+\left(v, x_{k}\right) \\
& =\left(e, x_{k+1}^{i_{k+1}} \cdots x_{p}^{i_{p}}\right)+(u, v)+\left(e, x_{k+1}^{j_{k+1}} \cdots x_{p}^{j_{p}}\right) \\
& \leqq(u, v)+2\left[\left(e, x_{k+1}\right)+\left(e, x_{k+2}\right)+\cdots+\left(e, x_{p}\right)\right] \\
& \leqq(u, v)+2\left(e, x_{k}\right)\left[\frac{1}{9}+\frac{1}{9^{2}}+\cdots+\frac{1}{9^{p-k}}\right] \\
& \leqq(u, v)+\left(e, x_{k}\right) / 4 .
\end{aligned}
$$

Therefore $(u, v) \geqq 3\left(e, x_{k}\right) / 4 \geqq 3\left(e, x_{n}\right) / 4 \geqq 3 d_{n}$. Consequently any two sets $C\left(n ; i_{1}, \cdots, i_{n}\right)$ of rank $n$ are separated by a distance at least equal to $3 d_{n}$, and condition ( $\mathrm{v})$ is satisfied.

Finally, $C\left(n ; i_{1}, \cdots, i_{n}\right)$ is totally bounded, since it is the union of a finite number $\left(2^{p-n}\right)$ of sets $C\left(p ; i_{1}, \cdots, i_{p}\right)$ having arbitrarily small diameter $d_{p}$. Any totally bounded closed subset of a complete space is compact, and therefore (i) is satisfied, provided $G$ is complete with respect to the metric $(x, y)$. If this is not the case, $G$ can be embedded isometrically in a complete space $\bar{G}$ and the same reasoning then shows that the closure of $C\left(n ; i_{1}, \cdots, i_{n}\right)$ in $\bar{G}$ is compact. Consequently, the sets $C\left(n ; i_{1}, \cdots, i_{n}\right)$ will be compact if they are all closed in $\bar{G}$. To ensure this, the sequence $\left\{x_{n}\right\}$, on which the construction rests, must be subjected to a further condition. Introduce a topologically equivalent metric $\rho(x, y)$ with respect to which $G$ is complete, and let the sequence $x_{1}, x_{2}, \cdots$ be so chosen that it satisfies not only condition (4.8) but also the condition

$$
\rho\left(a_{i_{1}} \ldots i_{n}, a_{i_{1}} \ldots i_{n} x_{n+1}\right) \leqq 2^{-n} \quad\left(i_{1}, \cdots, i_{n}=0,1\right) .
$$


Both conditions are satisfied by any sequence that converges to $e$ sufficiently rapidly. Condition (4.9) implies that for any sequence of subscripts $i_{1}, i_{2}, \ldots$ the sequence $a_{i_{1}} \ldots i_{n}(n=1,2, \cdots)$ is a Cauchy sequence with respect to the metric $\rho$, and therefore converges to an element of $G$. But any point of accumulation of $C$ in $\bar{G}$ is the limit of such a sequence. Hence $C$ is closed in $\bar{G}$, and likewise the sets $C\left(n ; i_{1}, \cdots, i_{n}\right)$. Thus in all cases we have constructed in $G$ a family of sets having properties (i) to (v).

Now consider any family of sets $C\left(n ; i_{1}, \cdots, i_{n}\right)$ satisfying conditions (i) to $(\mathrm{v})$. We proceed to define a measure such that $m C=1$. From condition (v) it follows that there exist positive numbers $\epsilon_{n}, \epsilon_{r} \rightarrow 0$, such that any two sets $C\left(n ; i_{1}, \cdots, i_{n}\right)$ of rank $n$ are separated by a distance at least equal to $d_{n}+2 \epsilon_{n}$. (In the family constructed above it suffices to take $\epsilon_{n}$ equal to $d_{n}$.) Consider the measuring system with $U_{n}$ equal to the $\epsilon_{n}$-neighborhood of $C(n ; 0, \cdots, 0)$, and $w_{n}=2^{-n}$. Then diam $U_{n} \leqq d_{n}+2 \epsilon_{n}$, hence diam $U_{n} \rightarrow 0$, and any set congruent to $U_{n}$ can overlap at most one set $C\left(n ; i_{1}, \cdots, i_{n}\right)$ of rank $n$.

To show that $m C=1$, consider any $r>0$ and choose $n$ such that diam $U_{n}<r$. From (iii) it follows that $C$ can be covered by $2^{n}$ sets congruent to $U_{n}$. Hence $L_{r}(C) \leqq 2^{n} w_{n}=1$. To establish the opposite inequality, consider any covering of $C$ by sets of the form $b_{i} U_{n_{i}}$. Since $C$ is compact, only finite coverings need be considered. Suppose $C \subset b_{1} U_{n_{1}} \cup b_{2} U_{n_{2}} \cup \ldots \cup b_{k} U_{n_{k}}$. Let $p$ be the largest of the indices $n_{1}, n_{2}, \cdots, n_{k}$ and let $q_{i}$ be the number of sets $C\left(p ; i_{1}, \cdots, i_{p}\right)$ of rank $p$ overlapped by $b_{i} U_{n_{i}}$. Since the sets $b_{i} U_{n_{i}}$ cover $C$ we must have $q_{1}+q_{2}+\cdots+q_{k} \geqq 2^{p}$. On the other hand, each set $b_{i} U_{n_{i}}$ can overlap at most one set of rank $n_{i}$, and therefore at most $2^{p-n_{i}}$ sets of rank $p$. Hence $2^{p-n_{i}} \geqq q_{i}$. Therefore $w_{n_{i}}=2^{-n_{i}}=2^{p-n_{i}} / 2^{p} \geqq q_{i} / 2^{p}$, and so $\sum_{i=1}^{k} w_{n_{i}} \geqq 2^{-p} \sum_{i=1}^{k} q_{i} \geqq 1$, which shows that $L_{r}(C) \geqq 1$. Thus $L_{r}(C)=1$ for every positive $r$. Therefore $m C=1$ and we have established the existence of a left-invariant Borel measure in $G$. This completes the proof of Theorem 3 in the separable case.

The structure of this measure within the unit set $C$ is easy to recognize. To each point $x \in C$ corresponds uniquely a sequence of indices $i_{1}, i_{2}, \ldots$ such that $x=\bigcap_{n} C\left(n ; i_{1}, \cdots, i_{n}\right)$. If we regard this sequence $\left\{i_{n}\right\}$ as an element of the infinite product group $C^{*}$ of the group of order two with itself, with the usual topology $[20$, p. 51$]$, it is not hard to see that this correspondence is a homeomorphism between $C$ and $C^{*}$, and that the measure $m$ in $C$ corresponds to the normalized Haar measure in $C^{*}$. Hence the measure $m$ in $G$ may be regarded as an extension of the measure in $C$ defined by this correspondence and the Haar measure in $C^{*}$.

The foregoing construction evidently contains a large degree of arbitrariness. In fact, it associates an invariant measure with any sequence $\left\{x_{n}\right\}$ that satisfies conditions (4.8) and (4.9). If a sequence satisfies these conditions, any subsequence will also satisfy them. In particular, the subsequence $\left\{x_{2 n}\right\}(n=1,2, \cdots)$ defines a unit set $C_{1} \subset C$ such that $m C_{1}=0$, where $m$ is 
the measure associated with the sequence $\left\{x_{n}\right\}$. Repeated application of this result gives rise to a sequence of measures $m_{1}, m_{2}, \cdots$, where $m_{k}$ is the measure associated with the subsequence $\left\{x_{k(n)}\right\}, k(n)=n \cdot 2^{k}$. These measures are all distinct and form an increasing sequence, $m_{1} A \leqq m_{2} A \leqq \cdots$ for every Borel set $\boldsymbol{A}$. It therefore appears that infinitely many invariant measures always exist, and there is certainly no uniqueness theorem for the problem of measure we have considered.

In case the group is locally compact, the construction may in some cases generate Haar's measure (see \$5). In the additive group of real numbers, however, this is certainly not the case, since conditions (i) to (v) are easily seen to imply that $C$ has Lebesgue measure zero. But in any case subsequences give rise to measures which are not locally finite, and the existence of such measures in any complete separable group is thus established.

5. An example. The preceding construction is illuminated by the following example, which also serves to illustrate some of the peculiarities that a measure may exhibit. Let $G$ be the complete infinite product group of the additive group of integers with itself or, equivalently, the additive group of integralvalued functions of a positive integer $n$. The elements of $G$ are sequences $x=\left(\xi_{1}, \xi_{2}, \cdots\right)$ of integers $\xi_{i}$. If $y=\left(\eta_{1}, \eta_{2}, \cdots\right)$ is any other element of $G$, define the distance $\rho(x, y)$ as $10^{-n}$, where $n$ is the least index such that $\xi_{n} \neq \eta_{n}$. It is easy to verify (see $\$ 10$ ) that $G$ is a complete separable group with respect to this metric, and that the metric is invariant. Not only is $G$ not locally compact, but no non-denumerable subgroup is locally compact.

Let $x_{n}$ be the sequence $\left\{\xi_{n i}\right\}$, with $\xi_{n i}=0$ for $i \neq n$ and $\xi_{n n}=1$. The sequence of elements $x_{1}, x_{2}, \ldots$ satisfies condition (4.8) and it is easy to follow through the construction, with the result that $C$ is the set of all elements $x$ with $\xi_{i}=0$ or 1 , and $U_{n}$ is the set of elements $x$ with $\xi_{1}=\xi_{2}=\cdots=\xi_{n-1}=0$, that is, the closed subgroup generated by $x_{n}, x_{n+1}, \ldots$. The resulting measure $m$ within $C$ corresponds to the Haar measure in the group $C^{*}$, as already noted. (Indeed we may identify $C^{*}$ with the set $C$, using the same metric but taking addition modulo 2 in each coordinate. The measure in $C^{*}$ generated by the sequence $x_{1}, x_{2}, \cdots$ is then equal to the normalized Haar measure in $C^{*}$. Consequently this is one group in which it is possible for the construction of $\$ 4$ to generate Haar's measure.)

Now consider the set $B C G$ consisting of all $x$ with $\xi_{i}=0$ or \pm 1 , that is, in additive notation, the set $C-C$. Using the invariance, and the fact that $m C=1$, one may show that $m B=+\infty$, and verify the following assertions:

(5.1) If $x \notin B$ then $(x+C) \cap C$ is void.

(5.2) If $x \in B$ and $\xi_{n} \neq 0$ for only $k$ indices $n$, then $m[(x+C) \cap C]=2^{-k}$.

(5.3) If $x \in B$ and $\xi_{n} \neq 0$ for infinitely many indices $n$ then $m[(x+C) \cap C]$ $=0$.

From these it follows at once that:

(5.4) $m[(x+C) \cap C] \leqq 1 / 2$ for $x \neq e$. 
This implies that the function $f(x)=m[(x+C) \cap C]$ is discontinuous at $x=e$. In fact it is discontinuous with respect to every possible topology in $G$ except the discrete topology. But the function $f(x)$ would have to be continuous if $m$ were a Haar measure [24, p. 41]. Consequently no topology in $G$ has $m$ for its corresponding Haar measure. We shall return to this remark later.

6. On the notion of completeness. A few remarks concerning the notion of completeness in groups may be in order. We have used the term "complete group" to mean a metrizable group that is complete with respect to some metric. Let us call this notion topological completeness. Van Dantzig [6] defines a group to be complete (komplett) if it has the property:

If $x_{n} x_{m}{ }^{-1} \rightarrow e$ then there exists an $x$ such that $x_{n} \rightarrow x$. An equivalent ( $\left.{ }^{4}\right)$ statement of this condition is the following:

If $x_{n}{ }^{-1} x_{m} \rightarrow e$ then there exists an $x$ such that $x_{n} \rightarrow x$. This is the same as requiring metric completeness with respect to some (and therefore every) left- (or right-) invariant metric.

For abelian groups the two kinds of completeness are equivalent (see below), but in general the condition of van Dantzig is stronger, as the following example shows. Let $G$ be the group of automorphisms (self-homeomorphisms) of the closed unit interval. This group is complete with respect to the metric $\rho_{1}(T, S)=\max |T x-S x|+\max \left|T^{-1} x-S^{-1} x\right|$, but not with respect to the (right-invariant) metric $\left(^{(5)} \rho_{2}(T, S)=\max |T x-S x|\right.$. Nevertheless these two metrics are topologically equivalent, since in both cases $T_{n} \rightarrow T$ means uniform convergence [22, p. 104]. Hence this group is topologically complete but not complete in the sense of van Dantzig. Moreover, it cannot be embedded in a complete (komplett) group. Indeed it can be asserted quite generally that for groups that can be completed in the sense of van Dantzig the two kinds of completeness are equivalent. For suppose $G$ is topologically complete and that it can be embedded as a dense subset of a "komplett" group $\bar{G}$. Then $G$ is a dense $G_{\delta}$ subgroup of $\bar{G}$ and therefore coincides $\left({ }^{(}\right)$with $\bar{G}$. Van Dantzig has given a necessary and sufficient condition that a group can be embedded in a "komplett" group, and this condition is satisfied by any abelian group. Moreover, any locally compact group is complete in both senses. Therefore

(4) Note however that the conditions $x_{n} x_{m}{ }^{-1} \rightarrow e$ and $x_{n}{ }^{-1} x_{m} \rightarrow e$ are not equivalent. See the example in footnote 5 .

(5) For example, the sequence of automorphisms $T_{n}$, where $T_{n}$ is the piecewise linear transformation that carries $0,1 / 2,1$ into $0,(2 n-1) / 2 n, 1$ respectively, is a Cauchy sequence in the second metric but not in the first. It also has the property that $T_{n} T_{m}{ }^{-1} \rightarrow I$ but $T_{n}{ }^{-1} T_{m}$ does not converge to $I$, where $I$ denotes the identity transformation. The latter property can also be expressed by saying that $T_{n}$ is a Cauchy sequence in the right uniform structure of $G$, but that $T_{n}^{-1}$ is not. This same example thus serves to answer a question raised by Bourbaki. See N. Bourbaki, Topologie générale. chap. 3, Actualités Scientifiques et Industrielles, no. 916, Paris, 1942, p. 28.

${ }^{(6)}$ Cf. Banach $[3$, p. 21, Theorem d]. See also the author's paper $[18$, p. 445] where it is shown that if $R$ is any dense $G_{\delta}$ subset of a complete group, then $R^{2}=G$. 
for such groups there is no distinction between the two kinds of completeness.

It therefore appears that after remetrization with a left-invariant metric a complete group may be incomplete with respect to the new metric, as remarked at the beginning of the proof of Theorem 3, and we see that whenever this complication arises it is unavoidable.

7. Extension of an invariant measure from a subset. We have observed that the measure constructed in $\$ 4$ can be regarded as an extension of a familiar measure within the unit set $C$, namely the measure corresponding to Haar's measure in the group $C^{*}$. Within the set $C$ the measure is invariant in the sense that congruent subsets of $C$ have equal measure. In this section we shall consider the general problem of extending an invariant measure from a subset, with a view to obtaining new methods of solving Problem 1. It should be noted that in the following theorem the group is not assumed to be complete or separable.

TheOREM $4\left({ }^{7}\right)$. Let $H$ be a Borel subset of a metric group $G$, and let $m$ be a Borel measure in $H$ with the property that $m(x A)=m A$ for every Borel set $A \subset H$ and $x \in G$ such that $x A \subset H$. There exist two left-invariant Borel measures $\lambda$ and $\nu$ in $G$, both of which are extensions of $m$, such that if $\mu$ is any other such extension of $m$ then $\lambda A \leqq \mu A \leqq \nu A$ for every Borel set $A \subset G$.

We shall call $\lambda$ and $\nu$ respectively the minimal and maximal extensions of $m$ from $H$.

We first define the maximal measure $\nu$. If $A$ is any subset of $G$ that can be covered by a sequence of sets congruent to $H$, define $\nu^{*} A=\inf \sum m H_{i}$, where the lower bound is taken for all sequences of Borel sets $H_{i} \subset H$ such that $A \subset \bigcup_{i} x_{i} H_{i}$ for some sequence of elements $x_{i} \in G$. For all other subsets of $G$, define $\nu^{*} A=+\infty$. This function $\nu^{*}$ is a left-invariant Carathéodory outer measure in $G$. The left-invariance of $\nu^{*}$, and properties (4.1), (4.2), and (4.3) may be verified at once. To verify (4.4) it is sufficient to show that if $\rho(A, B)>0$ then $\nu^{*} A+\nu^{*} B \leqq \nu^{*}(A \cup B)$. Let $G_{1}$ and $G_{2}$ be disjoint open sets such that $A \subset G_{1}$ and $B \subset G_{2}$. We may assume that $\nu^{*}(A \cup B)<+\infty$, and so there exist Borel sets $H_{i} \subset H$ and elements $x_{i} \in G(i=1,2, \cdots)$ such that $A \cup B \subset \bigcup_{i} x_{i} H_{i}$ and $\sum m H_{i}<\nu^{*}(A \cup B)+\epsilon$. Since $A \subset \cup_{i} x_{i}\left(x_{i}^{-1} G_{1} \cap H_{i}\right)$ and $B \subset \mathrm{U}_{i} x_{i}\left(x_{i}^{-1} G_{2} \cap H_{i}\right)$ it follows that

$$
\begin{aligned}
\nu^{*} A+\nu^{*} B & \leqq \sum\left[m\left(x_{i}^{-1} G_{1} \cap H_{i}\right)+m\left(x_{i}^{-1} G_{2} \cap H_{i}\right)\right] \\
& =\sum m\left[x_{i}^{-1}\left(G_{1} \cup G_{2}\right) \cap H_{i}\right] \leqq \sum m H_{i}<\nu^{*}(A \cup B)+\epsilon,
\end{aligned}
$$

which proves the desired inequality. Hence $\nu^{*}$ defines a left-invariant Borel measure $\nu$ in $G$, unless it assumes only the values 0 and $+\infty$.

To show that $\nu$ is an extension of $m$, and therefore that $\nu$ is finite and positive for at least one set, consider any Borel set $A \subset H$, and suppose that

$\left.{ }^{7}\right)$ Cf. Kolmogoroff [13], where an analogous theorem is proved. 
$A \subset \mathrm{Ux}_{i} H_{i}$, where $H_{1}, H_{2}, \cdots$ are Borel subsets of $H$. Then $A \subset \mathrm{Ux}_{i}\left(x_{i}^{-1} H \cap H_{i}\right)$, and since the sets $x_{i}\left(x_{i}^{-1} H \cap H_{i}\right)$ and $x_{i}^{-1} H \cap H_{i}(i=1,2, \cdots)$ are Borel subsets of $H$, it follows from the invariance property of $m$ that $m A \leqq \sum m x_{i}\left(x_{i}^{-1} H \cap H_{i}\right)=\sum m\left(x_{i}^{-1} H \cap H_{i}\right) \leqq \sum m H_{i}$. Hence $m A \leqq \nu A$. The opposite inequality is trivial. The maximal property of $\nu$ follows from the fact that if $\mu$ is any left-invariant Borel extension of $m$ to $G$, and if $A \subset U_{x_{i} H_{i}}$, then $\mu A \leqq \sum \mu\left(x_{i} H_{i}\right)=\sum m H_{i}$.

The minimal measure $\lambda$ is defined as follows. For any Borel set $A \subset G$, define $\lambda A=\sup \sum m H_{i}$, the upper bound being taken for all sequences of Borel sets $H_{i} \subset H$ such that for some sequence of elements $x_{i} \in G(i=1,2, \cdots)$ the sets $x_{i} H_{i}$ are disjoint and contained in $A$. It is evident that this is nonnegative set function and that it is left-invariant. To prove that it is countably additive, consider any sequence of disjoint Borel sets $A_{j} \subset G(j=1,2, \cdots)$ and let $A=\bigcup A_{j}$. It follows immediately from the definition that $\lambda A \geqq \sum \lambda A_{j}$. To establish the opposite inequality, suppose that $H_{1}, H_{2}, \cdots$ are Borel subsets of $H$, that $x_{i} H_{i} \cap x_{j} H_{j}$ is void if $i \neq j$, and that $U x_{i} H_{i} \subset A$. Define $H_{i j}=H_{i} \cap x_{i}{ }^{-1} A_{j}(i, j=1,2, \cdots)$. The sets $H_{i j}$ are evidently Borel subsets of $H$, for each $j$ the sets $x_{i} H_{i j}(i=1,2, \cdots)$ are disjoint subsets of $A_{j}$, and for each $i$ the sets $H_{i j}(j=1,2, \cdots)$ are disjoint sets whose union is $H_{i}$. From the definition of $\lambda$ and the additivity of $m$ it follows that $\sum_{i} m H_{i j} \leqq \lambda A_{j}$ and $\sum_{j} m H_{i j}=m H_{i}$. Hence $\sum_{i} m H_{i}=\sum_{i} \sum_{j} m H_{i j}=\sum_{j} \sum_{i} m H_{i j} \leqq \sum_{j} \lambda_{A_{j}}$, and therefore $\lambda A \leqq \sum_{j} \lambda A_{j}$.

If $A$ is a Borel subset of $H$, the inequality $\lambda A \geqq m A$ is trivial, and the opposite inequality follows immediately from the invariance of $m$ within $H$. Hence $\lambda$ is a left-invariant Borel extension of $m$ to $G$. The minimal property of $\lambda$ follows from the fact that if $\mu$ is any such extension of $m$, then for any sequence of disjoint Borel subsets of $A$ of the form $x_{i} H_{i}, H_{i} \subset H$, we have $\mu A \geqq \sum \mu\left(x_{i} H_{i}\right)=\sum m H_{i}$. This completes the proof of Theorem 4 .

If $m$ has the property that $m A=\sup m B$ for Borel sets $B \subset A$ with $m B<+\infty$, it is clear that $\lambda$ will also have this property.

The maximal and minimal measures are not in general equal, nor do they even have the same $\sigma$-finite contraction. For example, in the additive group of complex numbers, let $H$ be the subgroup of real numbers with ordinary linear measure $m$, and let $P$ denote the set of pure imaginary numbers. It is easy to verify that $\lambda P=0$ and $\nu P=+\infty$. Consequently the extension of a measure from a subgroup is not in general unique, though it is unique within each left-coset. It follows that a Borel measure is not in general determined uniquely by specifying a set of unit measure, in contrast to the situation in the case of Haar's measure in separable groups. As another example, consider the group of $\S 5$. The measure $m$ there defined is neither the maximal nor the minimal extension of a measure equal to $m$ within the set $C$. To see this, consider any countable dense set $A$ in $G$. By (4.7) there exists a dense $G_{\delta}$ set $A_{1} \supset A$ with $m A_{1}=0$. But from the definition of $\nu$.it is clear (in this instance) 
that every set of second category will have infinite measure. Hence $\nu A_{1}=+\infty$. On the other hand, let $A_{2}$ be the set of all $x$ in $G$ whose coordinates $\xi_{i}$ are all equal to 0 or 2 . Any set congruent to $C$ contains at most one point of $A_{2}$, and so $\lambda A_{2}=0$. But it is easy to verify that $m A_{2}=1$.

As a first application of Theorem 4 consider any locally compact metrizable group $G$ with Haar measure $m$, and take for $H$ an open neighborhood of unity with compact closure. It is easy to show that the maximal and minimal extensions of $m$ from $H$ are equal for all sets in the domain of $m$, indeed this follows from the uniqueness theorem. Moreover, $m$ is equal to the $\sigma$-finite contraction of $\lambda$ (but not in general of $\nu$ ). If the group is non-separable, the maximal and minimal extensions are certainly not equal for all Borel sets. Indeed, since the subgroup $G_{1}$ generated by $H$ is an open subgroup of $G$ with non-denumerably many cosets (because $G_{1}$ is separable), it follows that any set $A$ containing one and only one element of each left-coset of $G_{1}$ is closed and that $\lambda A=0$ and $\nu A=+\infty$. This justifies the remark made in $\S 3$ concerning the necessity of limiting the domain of Haar's measure. In a non-separable locally compact group it is not true that any two locally finite left-invariant Borel measures differ by a constant factor.

As a second application of Theorem 4 we can complete the proof of Theorem 3 , which was proved in $\$ 4$ only for separable groups. In a non-separable group one need only select a countable set that has at least one point of accumulation and take the closure of the subgroup generated by this set. The construction of $\$ 4$ applies to this subgroup, which is separable and complete, and then Theorem 4 can be used to extend the measure to $G$.

8. Special methods for defining invariant measures. In this section we shall consider some special methods for solving Problem 1. These methods, based on Haar's theorem, are somewhat more obvious than the construction used in $\$ 4$, but they are not as general.

THEOREM 5. If a metric group $G$ contains a locally compact subgroup $H$, dense in itself, then there exists a left-invariant Borel measure in $G$ which is an extension of Haar's measure in $H$.

To prove this, one need only take Haar's measure in $H$, or a Borel extension of it to $H$ in case $H$ is not separable, and then extend it to $G$. Theorem 4 applies since a locally compact subgroup is necessarily closed in $G$. In fact, the hypothesis can be weakened. It is sufficient to assume that $H$ is isomorphic to a locally compact group $H^{*}$, dense in itself, by a correspondence that makes Borel subsets of $H$ correspond to Borel sets in $H^{*}$. Then Haar's measure in $H^{*}$ defines a Borel measure in $H$ which can be extended to $G$.

It should be noted that in one respect Theorem 5 is more general than Theorem 3, since it does not require that $G$ be complete. But a complete group does not necessarily contain a locally compact subgroup which is dense in itself, as is shown by the example considered in $\$ 5$. Consequently the method 
of proof used here does not suffice to prove Theorem 3 in full generality.

In case $H$ is a normal subgroup of $G$, still another method is available for introducing an invariant measure in $G$. Let $\left\{U_{n}\right\}$ be a complete system of neighborhoods of $e$ in $G$, and let $V_{n}=U_{n} \cap H$. The sets $V_{n}$ satisfy the requirements for a system of neighborhoods of unity in a group. (According to Weil $[24$, p. 9], the following four properties characterize a system of neighborhoods $V$ in a group:

GT I. The intersection of the sets $V$ is $e$.

GT II. For each $V$ and $V^{\prime}$ there exists a set $V^{\prime \prime} \subset V \cap V^{\prime}$.

GT III. For each $V$ there exists a set $V^{\prime}$ such that $V^{\prime-1} V^{\prime} \subset V$.

GT IV. For each $x \in G$ and each $V$ there exists a set $V^{\prime}$ such that $V^{\prime} \subset x V x^{-1}$.

It is easy to verify that the sets $V_{n}$ satisfy these conditions.) Let $\bar{G}$ denote the group $G$ with the topology defined by the system of neighborhoods $\left\{V_{n}\right\}$. Within $H$, or any coset of $H$, the two topologies coincide, but in $\bar{G}$ the subgroup $H$ and its cosets are open as well as closed. Consequently $\bar{G}$ is locally compact, and any Borel set in $G$ is also a Borel set in $\bar{G}$. On the other hand, any Borel set in $\bar{G}$ that can be covered by countably many compact sets in $\bar{G}$ is a Borel set in $G$. Therefore the Haar measure in $\bar{G}$ defines a measure in $G$ whose minimal Borel extension is a Haar-Borel measure in the sense of the following definition.

Definition 3. A left-invariant Borel measure $m$ in a group $G$ is called a Haar-Borel measure if its $\sigma$-finite contraction is equal to the Haar measure in $G$ corresponding to some locally compact topology in this group.

We have proved the following theorem.

THEOREM 6. If a metric group $G$ contains a locally compact normal subgroup $H$, dense in itself, then there exists a Haar-Borel measure in $G$ which is an extension of Haar's measure in $H$.

As a matter of fact, the method just used to prove Theorem 6 can be subsumed under that used in proving Theorem 5 , for it is easy to see that it leads to the same measure as would be obtained by taking the minimal extension of the Haar measure in $H$. However, the minimal extension of a Haar measure from a subgroup which is not normal is not necessarily a Haar-Borel measure (see $\$ 12$, examples (b) and (c)), and so the conclusion of Theorem 6 is essentially stronger than that of Theorem 5.

In the proof of Theorem 6 the hypothesis that $H$ be normal was needed only to verify that the sets $V_{n}$ satisfy condition GT IV. For this purpose the following weaker hypothesis is sufficient (and also necessary). To each $x$ in $G$ corresponds a neighborhood $U$ of unity such that $U \cap H C x H x^{-1}$. Hence this condition on $H$ can be substituted for normality in the hypothesis of Theorem 6. No change is required in the proof except to replace "coset" by "left-coset." 
The special properties that distinguish Haar-Borel measures can be better understood after we have considered a somewhat more general class of measures studied by Weil, which we shall discuss in the next section.

9. Concerning Weil's converse of Haar's theorem. A. Weil [24, Appendix I] has proved the following converse of Haar's theorem. Given a group $G$ without a topology but with a left-invariant measure that satisfies certain conditions, one can use the measure to define a topology in $G$ such that $G$ can then be embedded as a dense subgroup of a locally compact group $\bar{G}$. Moreover, the given measure in $G$ corresponds to Haar's measure in $\bar{G}$ in the sense that any continuous function which vanishes outside a compact set in $\bar{G}$ has the same integral when integrated over $G$ by means of the given measure as when integrated over $\bar{G}$ by means of the Haar measure.

At first sight, this result appears to suggest that the problem of defining a left-invariant measure in any group is equivalent to the problem of defining a suitable topology in the group, and that every invariant measure is essentially a Haar measure. But we have seen ( $\$ 5$ ) that there exist invariant measures with properties quite unlike those of a Haar measure: To resolve this apparent contradiction we must examine more closely the assumptions underlying Weil's theory.

In the first place, Weil assumes that the given measure $m$ is $\sigma$-finite. Let the Borel field on which it is defined be denoted by $\mathfrak{I}$. Let $G \times G$ denote the direct product of $G$ with itself, and let $\mathfrak{I}_{2}$ be the least Borel field of subsets of $G \times G$ that includes all product sets of the form $A \times B$, where $A \in \mathfrak{T}$ and $B \in \mathfrak{T}$. Weil's further assumptions are as follows:

(M) The Borel field $\mathfrak{T}_{2}$ is invariant under the transformation $(x, y)$ $\rightarrow\left(y^{-1} x, y\right)$ of $G \times G$ into itself.

$\left(\mathrm{M}^{\prime}\right)$ For each $s \in G, s \neq e\left({ }^{8}\right)$, there exists a set $A \in \mathfrak{T}$ with finite positive measure, such that $m(A \cap s A)<m A$.

Of these assumptions, condition (M) is much the more important, and also the more difficult to understand. One might be tempted to think that any Borel measure would satisfy it; indeed if $\mathfrak{I}$ were the family of all Borel sets in $G, \mathfrak{I}_{2}$ would be the family of Borel sets in $G \times G$, and this family is invariant under the transformation in question. But since a Borel measure is not in general $\sigma$-finite, we must take for $\mathfrak{T}$ a proper subfamily of Borel sets, and when this is done the invariance of $\mathfrak{T}_{2}$ ceases to be evident. It turns out, in fact, that $\sigma$-finite contraction of a Borel measure may or may not satisfy (M).

The second assumption $\left(\mathrm{M}^{\prime}\right)$ is relatively unrestrictive. It is satisfied by any Borel measure in a separable metric group, since any neighborhood contains a set with positive finite measure.

For convenience we introduce the following definitions.

Definition 4. A left-invariant $\dot{\sigma}$-finite measure in a group $G$ is called a Weil measure if it satisfies conditions $(\mathrm{M})$ and $\left(\mathrm{M}^{\prime}\right)$.

${ }^{(8)}$ This condition is omitted from Weil's statement [24, p. 143], but is obviously intended. 
Definition 5. A left-invariant Borel measure in a topological group $G$ is called a Weil-Borel measure if its $\sigma$-finite contraction is a Weil measure.

Weil $[24, \S 11]$ has shown that any Haar measure is a Weil measure. Consequently, any Haar-Borel measure is a Weil-Borel measure.

The principal results of Weil's theory can now be stated more fully as follows. Given a group $G$ with a Weil measure $m$ defined on a Borel field $\mathfrak{T}$ of subsets of $G$, let $\mathfrak{T}_{0}$ denote the class of sets with finite positive measure. Then the sets $A A^{-1}, A \in \mathfrak{T}_{0}$, fulfill the requirements (GT I to GT IV of $\S 8$ ) for a system of neighborhoods of unity in a topological group, and thus define a topology in $G$. Moreover, in this topology there exists a "totally bounded" neighborhood of unity, that is, a neighborhood $U$ which can be covered by a finite number of sets congruent to $V$, where $V$ is an arbitrary neighborhood of unity. If $G$ is not already complete with respect to this topology, it can be completed to form a locally compact group $\bar{G}$ containing $G$ as a dense subgroup. The given measure in $G$ then corresponds to a Haar measure in $\bar{G}$ in the manner previously described. Instead of defining the topology by means of the sets $A A^{-1}$, it is shown that one may use an equivalent system of neighborhoods consisting of all sets $W(A, \epsilon), A \in \mathfrak{T}_{0}, \epsilon>0$, where $W(A, \epsilon)$ is the set of all $s \in G$ such that $m(A \cap s A) \geqq m A-\left(\epsilon^{2} / 2\right)$.

Returning now to the group considered in $\$ 5$, and the measure there defined, property (5.4) shows that if $\epsilon<1$ the set $W(C, \epsilon)$ contains only the element 0 . Hence the "topology" defined by these neighborhoods can only be the discrete topology. The corresponding Haar measure is not zero for points and therefore not equivalent to the given measure. This is not in accordance with Weil's conclusions, and so the Borel measure defined in $\$ 5$ cannot be a Weil-Borel measure. It follows that the $\sigma$-finite contraction of this measure must fail to satisfy condition (M), although it seems to be difficult to show this directly. Moreover, we shall show in $\$ 10$ that this group is one of a class of groups in which no Weil-Borel measure can exist. Consequently, the problem of defining an invariant Borel measure whose $\sigma$-finite contraction fulfills Weil's postulates is not in general soluble in groups which are not locally compact. This refinement of Problem 1, unlike those considered in Problem 2, is however soluble in some such groups, as is shown by Theorem 6.

10. A class of groups which admit no Weil-Borel measure. Let $\Gamma_{1}, \Gamma_{2}, \cdots$ be a sequence of groups each containing only countably many elements. Let $G$ be the set of all sequences $x=\left\{\xi_{i}\right\}$ with $\xi_{i} \in \Gamma_{i}(i=1,2, \cdots)$. If $x=\left\{\xi_{i}\right\}$ and $y=\left\{\eta_{i}\right\}$ are any two elements of $G$, define $x y=\left\{\xi_{i} \eta_{i}\right\}$. Then $G$ is a group with respect to this operation. Introduce a metric in $G$ by defining $\rho(x, y)=10^{-n}$ if $x \neq y$ and $n$ is the first index for which $\xi_{n} \neq \eta_{n}$, and define $\rho(x, x)=0$. With this metric $G$ becomes a metric group. A sequence $\left\{x_{k}\right\}$ of elements $x_{k}=\left\{\xi_{k i}\right\}$ of $G$ is a Cauchy sequence if and only if for each $i$ the sequence of $i$ th components $\xi_{1 i}, \xi_{2 i}, \xi_{3 i}, \cdots$ is constant from some term on. 
It is clear, then, that $G$ is complete with respect to this metric. It may be noted also that the metric is both left- and right-invariant. Furthermore, the subset $S$ consisting of all $x$ in $G$ with only a finite number of components different from unity is a countable dense set in $G$. Hence $G$ is a complete separable metric group. Furthermore, $G$ is zero-dimensional, since the spheres defined by $\rho(e, x) \leqq 10^{-n}$ are both closed and open. $G$ is compact if and only if all the groups $\Gamma_{i}$ are finite. $G$ is locally compact if and only if all but a finite number of the groups $\Gamma_{i}$ are finite. If infinitely many of the groups $\Gamma_{i}$ are infinite, then $G$ is nowhere locally compact, and in this case $G$ can be shown $\left(^{9}\right)$ to be homeomorphic to the set of irrational numbers. We shall call $G$ the complete direct product of the sequence of groups $\Gamma_{n}$.

Theorem 7. Let $G$ be the complete direct product of a sequence of countably infinite groups $\Gamma_{n}(n=1,2, \cdots)$ none of which contains an element of finite order. Then no Weil-Borel measure can exist in $G$.

To prove this, suppose that $m$ is a Borel measure in $G$ whose $\sigma$-finite contraction is a Weil measure. Let $\mathfrak{I}_{0}$ denote the class of sets $A$ with $0<m A<\infty$. Then, according to Weil, the sets $A A^{-1}, A \in \mathfrak{T}_{0}$, may be taken as neighborhoods of unity in a new topology, and in this topology there exists a neighborhood $U$ of unity which is such that it can be covered by a finite number of sets congruent to $V$, where $V$ is an arbitrary neighborhood of unity in the new topology. We shall obtain a contradiction by showing that this property cannot hold.

Since $m$ is a Borel measure in a complete separable space, Lemma 1 shows that an equivalent system of neighborhoods is provided by the sets $C C^{-1}$, where $C$ runs over all compact sets in $\mathfrak{I}_{0}$. (Throughout the present proof the term "compact" refers always to the original topology in $G$.) Hence we may assume that the neighborhood $U$ is of the form $U=C C^{-1}$. Let $V$ be a neighborhood of unity in the new topology such that $V^{3} \subset U$. We may assume that $V=C_{1} C_{1}^{-1}$, where $C_{1}$ is compact and in $\mathfrak{T}_{0}$, whence it follows that $V$ is compact.

Let $G_{n}$ denote the subgroup of $G$ consisting of all elements whose first $n-1$ components are equal to unity. (The sets $G_{n}$ constitute a complete system of closed open neighborhoods of unity in the original topology in G.) Consider the sets $V_{n}=V V^{-1} \cap G_{n}(n=1,2, \cdots)$. Evidently $V_{n} \supset V_{n+1}$, and $\bigcap_{n} V_{n}$ consists of unity alone. Furthermore, each of the sets $V_{n}$ is infinite; for if $x$ is any point of accumulation of the compact set $V$, then $e$ is a point of accumulation of $V x^{-1}$, and therefore $V x^{-1} \cap G_{n}$ is an infinite set contained in $V_{n}$. It follows that $V_{n+1}$ is a proper subset of $V_{n}$ for infinitely many indices $n$, say for $n_{1}<n_{2}<n_{3}<\cdots$. Thus, for each $k, V_{n_{k}}$ contains elements whose

(9) According to a theorem of Alexandroff and Urysohn [1, p. 95], any metric space which is complete, separable, zero-dimensional, and nowhere locally compact is homeomorphic to the set of irrational numbers. 
$n_{k}$ th component is different from unity. Let $B_{k}$ be the subset of $\Gamma_{n_{k}}$ consisting of all values assumed by the $n_{k}$ th components of elements of $V_{n_{k}}$. ( $B_{k}$ is the "projection" of $V_{n_{k}}$ on $\Gamma_{n_{k}}$.) Since $V_{n_{k}}$ is compact, $B_{k}$ is finite. Let $\alpha$ be an element of $B_{k}$ different from unity, and consider its successive squares $\alpha, \alpha^{2}, \alpha^{4}, \alpha^{8}, \cdots$. These elements are all distinct, since by hypothesis $\Gamma_{n_{k}}$ contains no element of finite order, and so only a finite number of them belong to $B_{k}$. Let $\beta$ denote the first element of the sequence whose successor does not belong to $B_{k}$, and let $x_{k}$ be an element of $V_{n_{k}}$ whose $n_{k}$ th component is equal to $\beta$. Then $x_{k}$ has the property that no element of $V_{n_{k}}$ has its $n_{k}$ th component equal to that of $y_{k}$, where $y_{k}$ denotes the square of the element $x_{k}$.

Now consider the sets $A_{n}=y_{n} V(n=1,2, \cdots)$. These sets are disjoint, for suppose $x \in A_{k} \cap A_{j}$, with $k<j$. Then $x=y_{k} v_{1}=y_{j} v_{2}$, where $v_{1} \in V$ and $v_{2} \in V$. Hence $y_{i}^{-1} y_{k}=v_{2} v_{1}^{-1} \in V V^{-1}$; and since the first $n_{k}-1$ components of $x_{k}$ and $x_{i}$ are equal to unity, we have also $y_{j}^{-1} y_{k} \in G_{n_{k}}$, and therefore $y_{j}^{-1} y_{k} \in V_{n_{k}}$. But since the $n_{k}$ th component of $x_{j}$ is equal to unity, the $n_{k}$ th component of $y_{j}^{-1} y_{k}$ is equal to that of $y_{k}$. This contradiction shows that the sets $A_{1}, A_{2}, \cdots$ are disjoint. But these sets are all congruent to $V$ and are contained in $V^{3}$. Consequently, $U$ contains infinitely many disjoint sets each congruent to $V$. It follows that $U$ cannot be covered by a finite number of sets congruent to $V$.

(To prove this last assertion, suppose $U \subset b_{1} V \cup b_{2} V \cup \ldots \cup b_{n} V$, and consider the sequence $y_{1}, y_{2}, y_{3}, \cdots$. These elements are all contained in $U$, since $e \in V$; and so at least two of them, say $y_{k}$ and $y_{j}$, are contained in the same set $b_{i} V$. Thus we have $y_{k}=b_{i} v_{1}$ and $y_{j}=b_{i} v_{2}$, whence $b_{i}=y_{k} v_{1}{ }^{-1}=y_{j} v_{2}-1$. Since $V=V^{-1}$ it follows that $b_{i} \in A_{k} \cap A_{j}$, contrary to the disjointness of these sets.)

In Theorem 7 , the assumption that the groups $\Gamma_{n}$ do not contain elements of finite order cannot be greatly relaxed. Indeed, if each group $\Gamma_{n}$ (or, at least, infinitely many of them) contains a finite normal subgroup $K_{n}$ of order at least 2, then $G$ contains a compact normal subgroup isomorphic to the complete direct product $K_{1} \times K_{2} \times \cdots$, and under these circumstances Theorem 6 shows that $G$ does admit a Weil-Borel measure. Consequently we can state the following theorem.

THEOREM 8. If $G$ is the complete direct product of a countable abelian group $\Gamma$ with itself infinitely many times, then in order that $G$ should admit no WeilBorel measure it is necessary and sufficient that $\Gamma$ contain no element of finite order.

It follows that in the group considered in $\$ 5$ no Weil-Borel measure can exist.

11. Various topologies in the group of real numbers. The additive group of real numbers, regarded as an abstract group $R$, is known to be uniquely characterized as an abelian group with the following three properties:

(11.1) $R$ contains no element of finite order. ( $R$ is torsion free.) 
(11.2) The equation $n x=a$ is soluble for arbitrary $a \in R$ and positive integer $n$. ( $R$ is divisible.)

(11.3) $R$ has the power of the continuum.

(The first two properties imply that division by a positive integer is universally possible and unique, therefore that the group admits the additive group of rationals as operators (cf. [8]). By the axiom of choice, there exists a Hamel basis [9] and the group is uniquely determined by the cardinal number of this set. The third condition ensures that any Hamel basis has the power of the continuum.)

Now, let $\Gamma$ be the additive group of rational numbers, and let $G$ be the complete direct product of $\Gamma$ with itself infinitely many times. It is easy to verify that $G$ has the three properties stated above, and Theorem 8 shows that $G$ admits no Weil-Borel measure. Hence it is possible to topologize the additive group of real numbers so that it becomes a complete separable non locally compact metric group with the property that it admits no Weil-Borel measure.

On the other hand, the additive group $H$ of vectors in Hilbert space likewise has properties (11.1) to (11.3). Any finite-dimensional linear manifold is a locally compact normal subgroup of $H$, and so determines a Haar-Borel measure in $H$, by Theorem 6 . In a similar way, the additive group of vectors in any finite-dimensional euclidean space $E_{n}$ is algebraically isomorphic to the group of real numbers. Each of these topologies defines a corresponding Haar measure in $R$, namely, the $n$-dimensional Lebesgue measure. Moreover, as Halmos [8] has remarked, the character group of the discrete additive group of rational numbers is an example of a compact group isomorphic to $R$. Likewise the direct product of this group with itself a finite or countably infinite number of times has the same property. As a matter of fact, from Pontrjagin's theory [21] of character groups it is not hard to show that all possible compact separable topologies in the additive group of real numbers are included in the sequence just described. For if $G$ is a compact separable group algebraically isomorphic to $R$, its character group $\Gamma$ is discrete, countable, torsion free, and divisible. Hence the number $\alpha$ of elements in a Hamel basis in $\Gamma$ is either finite or countably infinite. Accordingly, $\Gamma$ is isomorphic to the direct product of the rational group with itself $\alpha$ times. From the duality theorem for character groups it follows that $G$ is isomorphic to the character group of $\Gamma$. Moreover, the decomposition of $\Gamma$ into a direct product of rational groups corresponds to a decomposition of $G$ into a direct product of groups each isomorphic to the character group of the discrete rational group [21, Appendix I], and the dimensionality of $G$ is equal to $\alpha$. Thus $R$ can be given one and only one compact separable topology of dimension $\alpha$, where $\alpha$ is any positive

(10) This conclusion follows even more directly from another theorem of Pontrjagin [21, p. 387], according to which the character group of a discrete countable group $\Gamma$ is zero-dimensional if and only if all the elements of $\Gamma$ are of finite order. 
integer or $\infty$. But it cannot be given a compact zero-dimensional topology $\left({ }^{10}\right)$, that is, it cannot be given the topology of the Cantor set. (Any infinite topological group which is compact, separable, and zero-dimensional is homeomorphic to the Cantor set. For since it is homogeneous, it must be dense in itself; and the Cantor set is characterized, up to homeomorphism, by the properties of being compact, separable, zero-dimensional, and dense in itself.)

Thus we see that various topologies in the group of real numbers illustrate most of the conceivable possibilities. It can be given infinitely many distinct compact topologies, each with corresponding finite Haar measure; it can be given infinitely many distinct locally compact topologies, with corresponding infinite (but $\sigma$-finite) Haar measures; or it can be given a complete separable non locally compact topology that admits many distinct Haar-Borel measures, and also one that does not admit even a Weil-Borel measure. It is clear that no uniqueness theorem for Weil's measure in abstract groups is to be expected(11).

This example illustrates strikingly that the non-existence of a Weil-Borel measure in a given topological group in no way implies the non-existence of a Weil measure in the corresponding abstract group. The problem of determining what groups admit a Weil or Haar measure is not solved by the present investigation. In view of Weil's results, this problem is closely related to the problem of determining what groups admit a locally compact or locally totally bounded topology. What our investigation does show is that Problem 1 cannot be solved in general in this way.

12. Other examples. (a) Euclidean $n$-space $E_{n}$ regarded as an additive vector group. One may take the ordinary $k$-dimensional measure in a linear subspace $E_{k}(1 \leqq k<n)$ and extend it by Theorem 6 (or, equivalently, by taking its minimal extension $\lambda$ ) to obtain examples of Haar-Borel measures in $E_{n}$. The measures so defined will not be invariant under rotations of $E_{n}$, but certain other Borel extensions will be. For example, the $k$-dimensional measures defined by Hausdorff [10, p. 163] and Carathéodory [5] are invariant under any rotation or translation of $E_{n}$. However, unlike those considered above, these extensions of the measure in $E_{k}$ are not Weil-Borel measures. Indeed, no extension of the measure in $E_{k}$ can be invariant under rotation and also be a Weil measure. To see this, notice that $E_{k}$ can be rotated into a linear manifold $E_{k}^{\prime}$ which intersects $E_{k}$ in a manifold of lower dimension, and observe that Weil neighborhoods determined by subsets of $E_{k}$ and $E_{k}{ }^{\prime}$ intersect in sets of measure zero, contrary to the neighborhood axiom GT II.

Similar considerations apply to Hilbert space, where, for example, Cara-

(11) Weil proves a theorem [24, p. 148] to the effect that on the intersection of their respective domains of definition, two Weil measures in a group can differ at most by a constant factor, provided this intersection contains at least one set that has finite positive measure in both measures. This kind of conditional "uniqueness theorem" is of course not excluded by the above considerations. 
théodory's linear measure is a (non-Weil-) Borel measure invariant under any unitary transformation.

(b) The group of all self-homeomorphisms of the solid sphere. This is a complete separable group with respect to the uniform convergence topology (cf. §6). The subgroup of rotations about the center, or about a diameter, are compact. The minimal or maximal extensions of the Haar measures in these subgroups furnish examples of Borel measures in this group. None of these is a Weil-Borel measure, because each of these subgroups has a conjugate subgroup that intersects it only in the identity element, and consideration of Weil's neighborhoods shows that if a subgroup contains a neighborhood of the identity then any conjugate subgroup must also.

(c) The group $S_{\infty}$ of all permutations of the sequence of positive integers (see Schreier and Ulam [23]). If $f(n)$ and $g(n)$ are any two distinct permutations, define $\rho(f, g)=1 / n$, where $n$ is the least integer for which $f(n) \neq g(n)$ or $f^{-1}(n) \neq g^{-1}(n)$. This metric makes the group complete and separable, indeed homeomorphic to the set of irrational numbers. The closed subgroup generated by the permutations $(1,2),(3,4),(5,6), \ldots$ is a compact group isomorphic to the complete direct product of groups of order two, and therefore has a finite Haar measure which can be extended by Theorem 5. Again, let $n_{i, j}(i=1,2, \cdots ; j=0, \pm 1, \pm 2, \cdots)$ be an arrangement of the set positive integers as a doubly infinite array, and let $f_{i}$ denote the cyclic permutation of the sequence $\cdots, n_{i,-1}, n_{i, 0}, n_{i, 1}, \cdots$ defined by $f_{i}\left(n_{i, j}\right)=n_{i, j+1}$. Let $H$ be the closed subgroup generated by the permutations $f_{i}(i=1,2, \cdots)$. It is not hard to see that $H$ is topologically isomorphic to the group considered in $\$ 5$, and so the measure there defined determines a corresponding measure in $H$, which can then be extended by Theorem 4 . Neither of the above measures is a Weil-Borel measure. From our results, it is not clear whether or not a Weil-Borel measure can exist in $S_{\infty}$, but it can be shown that Theorem 6 fails to define one. For it is known [23] that no non-denumerable proper subgroup of $S_{\infty}$ is normal, consequently no subgroup can fulfill the hypotheses of Theorem 6.

\section{BIBLIOGRAPHY}

1. P. Alexandroff and P. Urysohn, Über nulldimensionale Punktmengen, Math. Ann. vol. 98 (1927) pp. 89-106.

2. S. Banach, On Haar's measure, in S. Saks, Theory of the integral, Warsaw and Lw6w, 1937, pp. 314-319.

3. - Théorie des operations linéaires, Warsaw, 1932.

4. C. Caratheodory, Vorlesungen ibber reellen Funktionen, Leipzig and Berlin, 1918.

5. - Über das lineare Mass von Punktmengen-eine Verallgemeinerung des Längenbegriffs, Nachr. Ges. Wiss. Göttingen, 1914, pp. 404-426.

6. D. van Dantzig, Zur topologischen Algebra I, Math. Ann. vol. 107 (1933) pp. 587-626.

7. A. Haar, Der Massbegriff in der Theorie der kontinuierlichen Gruppen, Ann. of Math. (2) vol. 34 (1933) pp. 147-169. 878 .

8. P. R. Halmos, Comment on the real line, Bull. Amer. Math. Soc. vol. 50 (1944) pp. 877- 
9. G. Hamel, Eine Basis aller Zahlen und die unstetigen Lösungen der Funktionalgleichung: $f(x+y)=f(x)+f(y)$, Math. Ann. vol. 60 (1905) pp. 459-462.

10. F. Hausdorff, Dimension und äusseres Mass, Math. Ann. vol. 79 (1919) pp. 157-179.

11. - Mengenlehre, 3rd ed., Berlin, 1935.

12. S. Kakutani, Über die Metrisation der topologischen Gruppen, Proc. Imp. Acad. Tokyo vol. 12 (1936) pp. 82-84.

13. A. Kolmogoroff, Beiträge zur Masstheorie, Math. Ann. vol. 107 (1933) pp. 351-366.

14. C. Kuratowski, Topologie, vol. 1, Warsaw and Lw6w, 1933.

15. L. H. Loomis, Abstract congruence and the uniqueness of Haar measure, Ann. of Math. (2) vol. 46 (1945) pp. 348-355.

16. D. Maharam, On measure in abstract sets, Trans. Amer. Math. Soc. vol. 51 (1942) pp. 413-433.

17. J. von Neumann, The uniqueness of Haar's measure, Rec. Math. (Mat. Sbornik) N.S. vol. 1 (1936) pp. 721-734.

18. J. C. Oxtoby, Note on transitive transformations Proc. Nat. Acad. Sci. U.S.A. vol. 23 (1937) pp. 443-446.

19. J. C. Oxtoby and S. M. Ulam, On the existence of a measure invariant under a transformation, Ann. of Math. (2) vol. 40 (1939) pp. 560-566.

20. L. Pontrjagin, Topological groups, Princeton, 1939.

21. - The theory of topological commutative groups, Ann. of Math. (2) vol. 35 (1934) pp. $361-388$.

22. J. Schreier and S. Ulam, Über topologische Abbildungen der euklidischen Sphären, Fund. Math. vol. 23 (1934) pp. 102-118.

23. — Über die Permutationsgruppe der natïrlichen Zahlenfolge, Studia Mathematica vol. 4 (1933) pp. 134-141.

24. A. Weil, L'integration dans les groupes topologiques et ses applications, Actualités Scientifiques et Industrielles, no. 869, Paris, 1940.

Bryn Mawr College, Bryn Mawr, Pa. 